\title{
Fully Automatic Detections of Abnormalities of Brain MR Images by utilizing Spatial Information and Mathematical Morphological Operators
}

\author{
Arshad Javed $^{1,2, *}$, Wang Yin Chai ${ }^{1}$, Abdulhameed Rakan Alenezi ${ }^{2}$ and Narayanan Kulathuramaiyer ${ }^{1}$ \\ ${ }^{1}$ Faculty of Computer Science \& Information Technology, Universiti Malaysia Sarawak, 94300 Kota Samarahan, Sarawak, Malaysia \\ ${ }^{2}$ Faculty of Computer Science \& Information, Aljouf University, P. O. Box 2014 , Sakaka, Saudi Arabia
}

Received: 27 Mar. 2014, Revised: 27 Jun. 2014, Accepted: 28 Jun. 2014

Published online: 1 Jan. 2015

\begin{abstract}
Image segmentation refers to the process of partitioning a digital image into multiple sets of pixels are known as segments. The main goal of image segmentation is to change and simplify the representation of an image into something that is more meaningful and easier to analyze. The manual transactions for segmentation by experts is a difficult phenomena and time consuming process as well as. Most of the images in the process received are lacking of good quality. The main objective of this study is to develop a reliable mechanism to enhance the image quality and extract the abnormal portion through brain MR image accurately. A spatial filter is designed by utilizing the spatial information of the image and further to use collective information to enhance the poor quality of image(s), whereas, k-means clustering and mathematical morphological operations which extract the tumor segment from images. The proposed method is applied on different types of brain MR images for both visual and quantitative evaluations. Experimental results concluded during the practicum showed promising and reliable accuracy to open a thorough research for better future perspective of the technique developed in the article.
\end{abstract}

Keywords: Image segmentation, Morphological operators, Spatial mask

\section{Introduction}

In recent days, brain tumor is becoming a dreadful disease and spreading rapidly in all human societies throughout the world because of changing trends in human activities towards adopting unnatural and artificial means of life. This is causing to develop depressions which is supportive to brain tumors and if not diagnosed at an early stage in time can lead to colossal damage of body and brain and even to meet uncertain deaths before time in all ages of human beings. Medical reports and statistics of last few years show that brain tumor is becoming one of the alarming lethal disease and the experts have opinion that in future if not controlled, brain tumor might be one of the increasing disease for death rates.

It has been realized that one who suffers in brain tumor disease live in a complete paralyzed situation. Recovering such a person into normality demands us to pay him proper attentions through medical care and utilizing technical resources. Medical Experts use manual diagnostic method of segmentation of brain MR images which is very expensive, time consuming and non-repeatable process and this method although fulfills the needs but there are apprehensions of interpreting errors. For this purpose, there is an attempt in this article to design computer-aided diagnostic system which would help the experts to take a second opinion from the system which we are going to introduce below. Image segmentation is the process of splitting up an image into significant regions of particular problem which is required to be focused. It pertains with properties like gray level, color, shape, textures etc., help to recognize regions and distinguishing similarities. It then forms a spectrum and cluster very rarely possessing of ambiguity and more often with utmost clarity for building of groups of regions where each such group having a particular meaning to acquire result oriented accuracy for the physicians of this horrific disease.

On the other hand segmentation is the process of extracting points, lines or regions which are then used as

\footnotetext{
* Corresponding author e-mail: arsh_qau@ju.edu.sa
} 